\title{
Aus der Sicht des Patienten
}

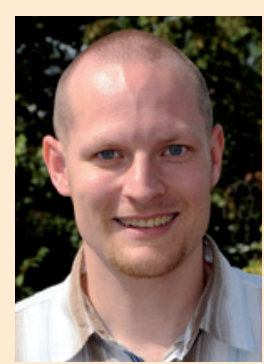

Christian Schäfer, Stuttgart
Warum soll man eigentlich Transplantationszentren und Ärzten weiterhin Anreize für möglichst viele transplantierte Organe geben? Wäre es nicht viel besser, eine hohe Ergebnisqualität bzgl. des Patientenüberlebens und der Organfunktion zu belohnen? Diese berechtigten Fragen stellte die Deutsche Gesellschaft für Nephrologie (DGfN) im Februar in den Raum. Sie greift damit die nach wie vor schwierige Situation in der deutschen Transplantationsmedizin auf: Die Bedürfnisse des Patienten stünden nicht im Mittelpunkt, denn dieser wünsche sich nicht, dass eine Einrichtung möglichst viele Organe transplantiert, sondern dass er mit dem transplantierten Organ möglichst lange leben kann. Das ist einleuchtend! Daher ist die Forderung der DGfN nach einer Ausrichtung der Anreize auf die Ergebnisqualität sehr vernünftig.

Ein weiterer Appell der DGfN an die Politik (insbesondere an den Bundesgesundheitsminister Hermann Gröhe) ist die Einrichtung eines Bundestransplantationszentrums (BTZ). Mit solch einem überregionalen Zentrum unter der Aufsicht des Bundesgesundheitsministeriums könne man die Initiative verschiedener Bundesländer und Transplantationszentren, den Patienten und ein optimales Ergebnis der Transplantation in den Vordergrund zu stellen, unterstützen. Somit könnten auch der Aufbau des geplanten bundesweiten Transplantationsregisters und die Verabschiedung von Richtlinien in einer Behörde - dem BTZ - vereint werden. Konflikte wegen Kompetenzzuweisungen müsste man noch lösen: So ist derzeit etwa die Bundesärztekammer (BÄK) für die Richtlinien zuständig. Dies ist allerdings etwas, das mit Kompromissen im Dienste der Sache lösbar ist. Das BTZ wäre dann eine Körperschaft des öffentlichen Rechts - im Gegensatz zur Deutschen Stiftung Organtransplantation (DSO), die nur zum Teil öffentlich-rechtlich ausgerichtet ist. Dies könnte einen größeren Schritt in Richtung Vertrauensrückgewinn der Bevölkerung in die Organspende bzw. -vergabe bedeuten, als es die Neukonstitution der DSO im August 2013 war: Damals bewegte man sich vom privatrechtlichen Charakter hin zu einer stärker öffentlich-rechtlichen Ausrichtung. Die Organspendezahlen blieben in Deutschland aber leider weiterhin auf einem niedrigen Niveau, sodass hier tatsächlich ein dringender und grundlegender Handlungsbedarf besteht - v.a. aus der Sicht der Patienten auf der Warteliste.

Wäre es angesichts dieser unbefriedigenden Situation nicht schön, wenn es Möglichkeiten gäbe, z.B. eine Niere „nachwachsen“ zu lassen, damit man nicht auf ein Spenderorgan angewiesen ist? Oder wenn wir auf verbesserte Dialyseregime und -verfahren sowie tragbare Dialysegeräte zurückgreifen könnten, damit die Patienten den Lebensabschnitt an der Dialyse besser und komplikationsärmer verbringen können? Um einmal den aktuellen Stand zu diesen interessanten Fragestellungen für Sie zusammenzufassen, haben wir den Schwerpunkt der vorliegenden Ausgabe der Dialyse aktuell auf das Thema „Neue Therapieverfahren“ gelegt. Ich wünsche Ihnen viel Erkenntnisgewinn bei der Lektüre der Beiträge! 